
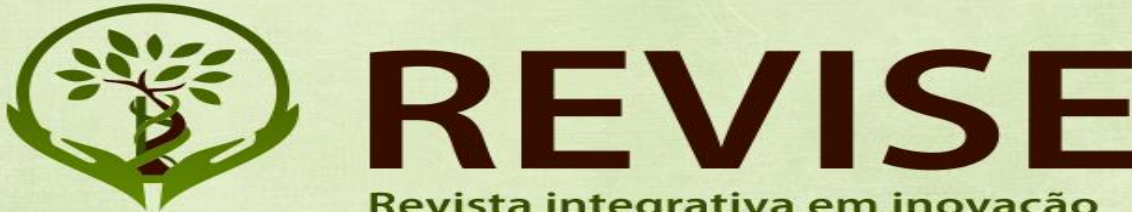

Revista integrativa em inovação tecnológica nas ciências da saúde

\title{
SEGURANÇA ALIMENTAR E NUTRICIONAL EM PROGRAMA DE ALIMENTAÇÃO: APROXIMAÇÕES E DISTÂNCIAS ENTRE BASES NORMATIVAS DE INSTITUCIONALIZAÇÃO.
}
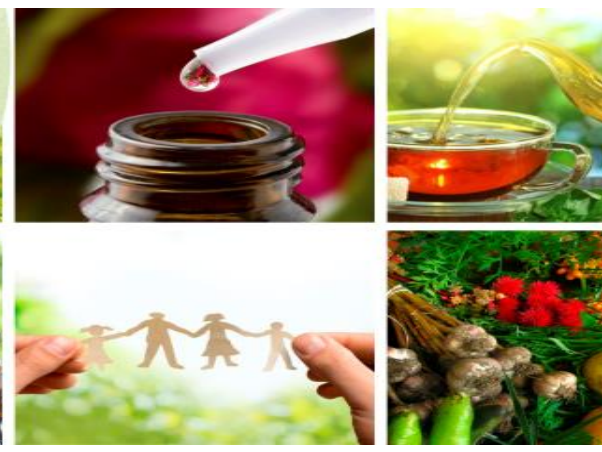

\author{
Lívia Silva dos Santos \\ Flávia Conceição dos Santos Henrique \\ CCS UFRB
}

\section{RESUMO}

Numa versão idealizada, consolidando princípios a Segurança Nutricional e Alimentar SAN, seria considerada como condição em que todas as pessoas em todos os lugares, teriam garantido acesso a alimento de qualidade, em quantidade o suficiente para atender as necessidades biológicas do indivíduo. A SAN traz com ela algumas Políticas intersetoriais, podendo citar o Programa Nacional de Alimentação Escolar (PNAE), que visa suprir parcialmente as necessidades nutricionais dos alunos, e a Política Nacional de Alimentação e Nutrição (PNAN) que tem o propósito de garantir a qualidade dos alimentos para consumo no país, além de promover práticas saudáveis. Objetiva-se analisar o discurso - aproximações e distanciamentos - da SAN no âmbito de dois programas setoriais de alimentação e nutrição. Utilizou-se do método de análise documental, e explorou a base normativa do programa e políticas de referência para o estudo. Apesar dessas políticas terem tido um crescimento, e sofrido transformações para suas melhorias, pode-se observar que ainda há necessidade de dar continuidade a esse processo. Observou-se que a maioria dos documentos avaliados, trazem consigo trechos onde afirmam que as políticas intersetoriais estão inseridas no contexto da SAN. Palavras-Chave: Segurança Alimentar e Nutricional; Alimentação Escolar; Política Nacional de Alimentação.

\begin{abstract}
In an idealized version, consolidating the principles of Nutrition and Food SecuritySAN, would be considered as a condition in which all people everywhere would have guaranteed access to quality food in enough quantity to meet the biological needs of the individual. The SAN brings with it some intersectoral policies and may mention the National School Feeding Program (PNAE), which aims to partially meet the nutritional needs of students, and the National Food and Nutrition Policy (PNAN) which aims to ensure quality. consumption in the country, as well as promoting healthy practices. The objective is to analyze the discourse - approximations and distances - of the SAN within two sectoral programs of food and nutrition. We used the document analysis method and explored the normative basis of the program and reference policies for the study. Although these policies have grown and undergone transformations for their improvement, it can be observed that there is still a need to continue this process. Most of the documents evaluated present excerpts where they state that intersectoral policies are inserted in the context of the FNS.

Keywords: Food and Nutrition Security; School feeding; National Food Policy
\end{abstract}

Segurança alimentar. Revista Revise, vol 3, $n^{o}$ fluxo contínuo, p.46-63. 

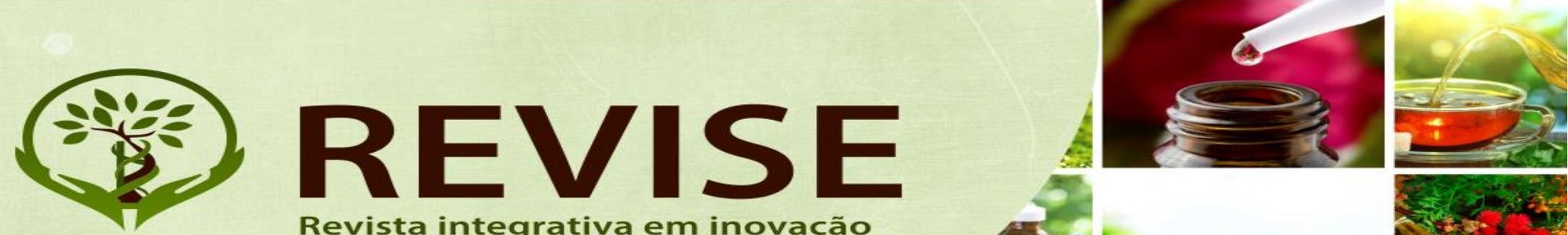

Revista integrativa em inovação tecnológica nas ciências da saúde

\section{INTRODUÇÃO}

A Segurança Alimentar e Nutricional (SAN) é um tema presente na agenda pública nacional há aproximadamente 45 anos, inicialmente como possibilidade de enfrentamento de uma crise alimentar e, na medida de sua apropriação pela sociedade civil organizada, sobretudo, como conceito amplo capaz de garantir o combate à fome e a garantia do direito humano à alimentação adequada e saudável. Tal como expresso na Lei Orgânica, a SAN pode ser entendida como a realização do direito de todos a alimentos de qualidade, em quantidade suficiente, e de modo que não venha afetar outras necessidades. Este conceito de SAN abarca aspectos muito amplos, como: a qualidade dos alimentos, condições para produção, o desenvolvimento sustentável e a qualidade de vida da população. Dessa forma, o Sistema de Segurança Alimentar e Nutricional (SISAN) tem como princípios a universalidade, intersetorialidade, participação social, e equidade (social, econômica, étnica e de gênero) (BRASIL, 2006).

Pela complexidade e amplitude do conceito, pelos princípios e objetivos incorporados à LOSAN, a SAN como objetivo estratégico nacional, deve articular e permear um conjunto de políticas e ações de governo, comprometendo diferentes segmentos sociais em parceria. Para Prado et al (2010), como resultado do tratamento dado à questão alimentar e nutricional, o direito humano deve ser atendido a partir de referenciais éticos e envolvendo questões estruturais, como o enfrentamento às desigualdades sociais e econômicas. Esse desafio de ofertar alimentação adequada a todos, abarca uma série de políticas públicas. Dentre essas, duas das mais estruturantes, remetem aos setores saúde e educação e referem-se, respectivamente à Política Nacional de Alimentação e Nutrição (PNAN) e ao Programa Nacional de Alimentação Escolar (PNAE).

A PNAN foi aprovada em 1999, onde o Estado, junto à políticas públicas, compromete-se em promover, proteger e prever os direitos básicos humanos à qualidade, utilização biológica da alimentação e saúde, ou seja, tem o propósito de

Segurança alimentar. Revista Revise, vol 3, no fluxo contínuo, p.46-63. 

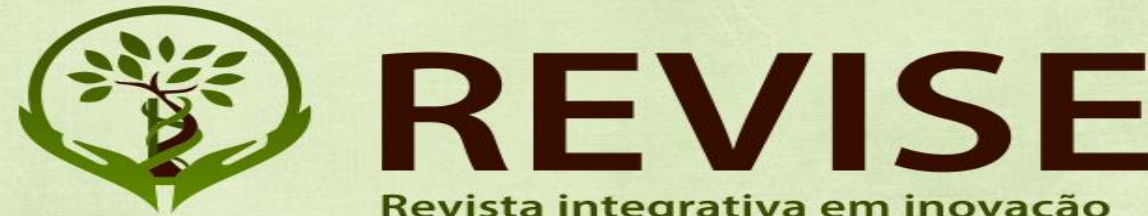

Revista integrativa em inovação tecnológica nas ciências da saúde

ISSN: $2179-6572$

garantir a qualidade dos alimentos para o consumo no país, bem como promover hábitos alimentares saudáveis, controlando assim distúrbios alimentares. A Política tem algumas diretrizes como, estímulo a ações intersetoriais com vistas ao acesso universal aos alimentos; garantia da qualidade e segurança dos alimentos; monitoramento da situação nutricional e alimentar.

Já o PNAE, implantado em 1955, foi assinado um decreto que institui a Campanha da Merenda Escolar (CME), e somente em 1979, após sofrer várias transformações, passou então a denominar-se PNAE, a qual, busca "contribuir para o crescimento, o desenvolvimento, a aprendizagem, o rendimento escolar dos estudantes e a formação de hábitos alimentares saudáveis por meio da oferta da alimentação escolar e de ações de educação alimentar e nutricional.” (BRASIL,2012). O PNAE tem como objetivo, oferecer uma alimentação adequada para todos da atenção básica, ou seja, desde a educação infantil à educação de jovens adultos. Essa cobertura, é garantida aos alunos de escolas públicas, via repasse do Fundo Nacional do Desenvolvimento da Educação (FNDE), para as prefeituras municipais, secretarias de educação dos estados e do Distrito Federal, creches, pré-escolas e escolas federais (BRASIL, 2010).

Em que pese a PNAN e o PNAE, em contexto amplo, estarem inseridos no SISAN, como estratégias setoriais de promoção da SAN, sabe-se que foram formulados em momento anterior à sanção da LOSAN e em diferentes contextos político-sociais e institucionais. Nesse sentido, emerge a questão sobre as aproximações e distanciamentos entre o conceito de SAN expresso na LOSAN e aqueles expressos nos marcos normativos desses dois programas, tão estratégicos à promoção da SAN.

Nessa perspectiva o estudo traz como questão norteadora: Qual o discurso de SAN no âmbito de programas de alimentação e nutrição? Partimos do pressuposto que o arcabouço jurídico-normativo, enquanto dimensão material, é a principal referência à implementação de políticas públicas e responde, em grande medida, pelo sucesso ou fracasso de intervenções nas esferas locais uma vez que se constitui na principal referência às gestões. Compreendemos que, à medida que a SAN se realiza a partir de

Segurança alimentar. Revista Revise, vol 3, no fluxo contínuo, p.46-63. 

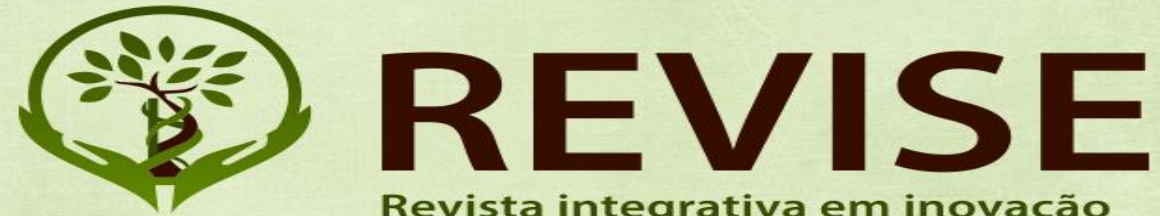

Revista integrativa em inovação tecnológica nas ciências da saúde

ISSN: $2179-6572$

diversas intervenções setoriais, os diferentes discursos expressos nas bases normativas favorecem ou dificultam o entendimento sobre a política ou programa, seus objetivos e modos de realização.

Buscando responder ao questionamento proposto, o objetivo geral do estudo foi analisar o discurso - aproximações e distanciamentos - da Segurança Alimentar e Nutricional no âmbito de dois programas setoriais de alimentação e nutrição.

\section{MÉTODO}

O presente estudo utilizou-se do método de análise documental, e explorou a base normativa do programa e políticas de referência para o estudo obtidos via internet, em sites institucionais. Abarcou a integração de documentos (fontes secundárias) e, ainda, trabalhos sobre o tema definido. Esses documentos são capazes de fornecer dados atuais e relevantes em relação à temática em questão, compreende toda bibliografia tornada pública com o intuito de demonstrar ao pesquisador tudo aquilo que já foi publicado sobre determinado assunto (MARCONI; LAKATOS, 2009).

A pesquisa foi iniciada a partir da questão norteadora formulada: Qual o discurso de SAN no âmbito de programas de alimentação e nutrição?

Por se tratar de pesquisa documental, o aprofundamento na leitura orientou a incorporação de referências teóricas complementares para facilitar o alcance dos objetivos propostos. Buscou-se, portanto, na literatura textos que complementassem os encontrados, no sentido de enriquecer a exploração do tema proposto. Para a investigação e busca de documentos foi realizada uma pesquisa na base de dados do Scielo, através das terminologias adotadas pelos Descritores em Ciências da Saúde (DeCS), foi realizada uma busca simples, utilizando o descritor "Segurança Alimentar e Nutricional", sendo encontrados 166 documentos. Posteriormente, para melhor filtrar os documentos, realizou-se uma busca avançada com os descritores: Segurança alimentar e nutricional no Brasil; PNAN e PNAE.

Segurança alimentar. Revista Revise, vol 3, no fluxo contínuo, p.46-63. 

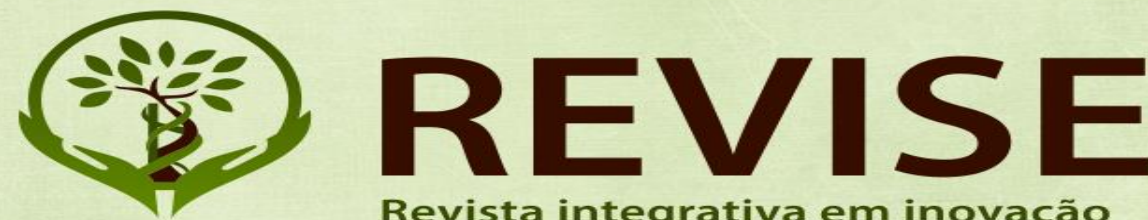

Revista integrativa em inovação tecnológica nas ciências da saúde

A fim de fazer outras buscas investigativas foi realizada uma pesquisa na base de dados Virtual em Saúde (BVS). Através das terminologias adotadas pelos Descritores: em Ciências da Saúde (DeCS), foi realizada uma busca simples, utilizando o descritor "Segurança Alimentar e Nutricional”, sendo encontrados 201 documentos. Posteriormente, para melhor filtrar os documentos, realizou-se uma busca avançada com os descritores "Segurança alimentar e nutricional no Brasil; PNAN e PNAE.” na qual foram encontrados 83 sendo que alguns já tinham sido encontrados anteriormente no (Scielo).

Ao realizar a pesquisa na base de dados, e utilizando-se dos critérios de inclusão estabelecidos, foram selecionados 34 documentos, entre artigos e bases normativas. Após esse levantamento bibliográfico, realizou-se uma leitura flutuante do material selecionado. Assim, pôde-se obter uma visão global do material, encontrado para a pesquisa. Em seguida, efetuou-se a leitura exploratória, a qual permitiu um melhor entendimento sobre a temática.

Para a análise dos dados, foi utilizada a técnica da análise de conteúdo de Minayo (2010), que compreende três etapas: pré-análise ou momento de ordenação de dados e documentos que serão utilizados na pesquisa de acordo com as hipóteses e objetivos do estudo; Exploração do material ou fase de codificação e agregação dos dados obtidos.

\section{Tratamento dos resultados e interpretação dos dados}

De acordo com Bardin e Minayo (1979), essas etapas compreendem: A pré análise é fase de organização e sistematização das ideias, em que ocorre a seleção das informações a serem avaliadas, a retomada das hipóteses e dos objetivos iniciais da pesquisa em relação ao material coletado, e a elaboração de indicadores que orientarão a interpretação final. Quanto à exploração do material trata-se da etapa em que os dados brutos são codificados para se alcançar o núcleo de compreensão do texto. A 

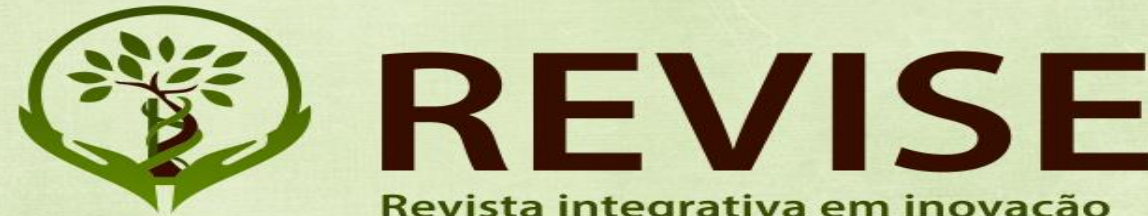

Revista integrativa em inovação tecnológica nas ciências da saúde

ISSN: 2179-6572

codificação envolve procedimentos de recorte, contagem, classificação, desconto ou enumeração em função de regras previamente formuladas. Os resultados obtidos, aliados ao confronto sistemático com o material e às inferências alcançadas, podem servir a outras análises baseadas em novas dimensões teóricas ou em técnicas diferentes.

\section{RESULTADOS E DISCUSSÃO}

\subsection{Segurança Alimentar e Nutricional (SAN): um breve histórico}

A Constituição Brasileira de 1988 refere-se à saúde como resultante de diversas condições, sendo elas, alimentação, educação, trabalho, renda, acesso aos serviços de saúde, dentre outras, devendo ser um direito garantido por meio da implantação de políticas econômicas e sociais, que devem promover a integração de diversos setores de governo. Com isso, os países têm a soberania de decidir o que produzir para quem produzir e como produzir. Essa soberania dará direito à cada país a elaborar as suas estratégias para a produção, consumo e políticas. Esta abrange a valorização das culturas locais e regionais (BRASIL, 2010).

Em 1994, foi realizada a I Conferência Nacional de Segurança Alimentar, a qual, teve como objetivo discutir as diretrizes para a preparação de uma Política Nacional de Segurança Alimentar e Nutricional (PNSAN), e também expandiram as discussões sobre a promoção dos Direitos Humano, Econômicos, Sociais e Culturais (DHESC), entre eles o DHAA (BRASIL, 2008).

Com relação á segurança alimentar e nutricional, em de 15 de setembro de 2006, cria-se o Sistema Nacional de Segurança Alimentar e Nutricional (SISAN), instituído pela Lei Orgânica de Segurança Alimentar e Nutricional de $\mathrm{n}^{\circ} 11.346$, com vistas a assegurar o Direito Humano à Alimentação Adequada (DHAA) e dá outras providências envolvendo a integração entre diferentes setores governamentais (CUSTÓDIO, et al, 2011). Tal como expressa a LOSAN (BRASIL, 2006), a SAN é entendida como:

“A realização do direito de todos ao acesso regular e permanente a alimentos de qualidade, em quantidade suficiente, sem comprometer o acesso a outras

Segurança alimentar. Revista Revise, vol 3, no fluxo contínuo, p.46-63. 


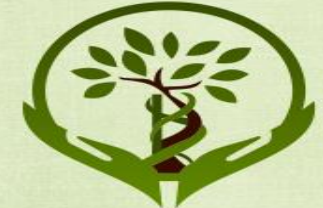

ISSN: 2179-6572

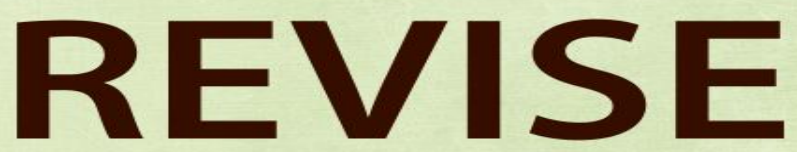

Revista integrativa em inovação tecnológica nas ciências da saúde

necessidades essenciais, tendo como base práticas alimentares promotoras de saúde que respeitem a diversidade cultural e que sejam ambiental, cultural, econômica e socialmente sustentáveis". (Brasil, 2010, p 14)

Ao falar de Segurança Alimentar e Nutricional, abre-se um leque envolvendo vários aspectos de oferta (produção, desenvolvimento sustentável, abastecimento e comercialização), e de demanda (aproveitamento e aquisição) de alimentos. O conceito de Segurança Alimentar emerge na Europa, no início do século XX, onde passou a se pensar que cada país poderia produzir seu próprio alimento, evitando vulnerabilidades, e adquiriu uma perspectiva internacional com a criação da Organização das Nações Unidas para a Agricultura e a Alimentação (CUSTODIO, 2009).

O conceito de Segurança Alimentar passou a ser disseminado internacionalmente após a Conferência Mundial de Alimentação de 1974, se afastando do foco original das Nações Unidas, e no decorrer dos anos, mais especificamente em 1980, voltou-se para a questão somente do acesso (demanda e distribuição), passando para segundo plano a da oferta de alimentos (BRASIL, 2011).

A dimensão nutricional passou a fazer parte com mais força no conceito de Segurança Alimentar no final dos anos 80; em 1992 a FAO passou a novamente ressaltar a importância da qualidade nutricional, sanitária, biológica e cultural dos alimentos para a SAN. Em 1996, algumas ideias derivadas da I CNSA realizada em 1994, deram origem a base do relatório sobre a situação da Segurança Alimentar e da Fome no Brasil. Este documento foi entregue, pelo governo brasileiro, à Cúpula Mundial de Alimentação, em Roma, por uma comissão tripartite que era formada pelo governo, iniciativa privada e sociedade civil. Neste documento a SAN passou a ser conceituada da seguinte forma: Segurança Alimentar e Nutricional consiste em garantir a todos condições de acesso a alimentos básicos, seguros e de qualidade, em quantidade suficiente, de modo permanente e sem comprometer o acesso a outras necessidades essenciais, com base em práticas alimentares saudáveis, contribuindo assim para uma

Segurança alimentar. Revista Revise, vol 3, no fluxo contínuo, p.46-63. 

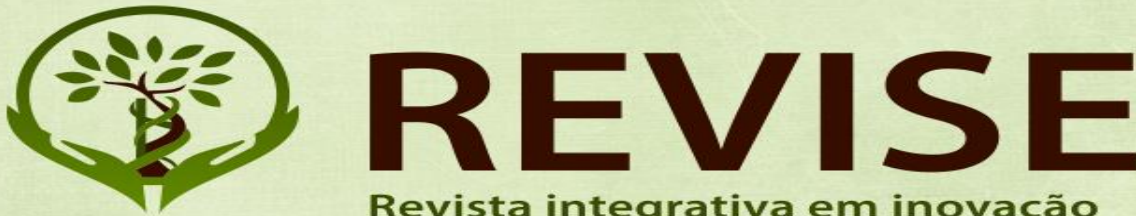

Revista integrativa em inovação tecnológica nas ciências da saúde

ISSN: 2179-6572

existência digna em um contexto de desenvolvimento integral da pessoa humana (BRASIL, 1996, p. 4).

Ainda em 1996, com a realização da Cúpula Mundial de Alimentação, os chefes de Estado e de Governo garantiram o direito dos homens de terem acesso a alimentos seguros e nutritivos. O compromisso principal era garantir um ambiente político, social e econômico, destinado a criar as melhores condições para erradicar a pobreza e que favoreça ao máximo a realização de uma segurança alimentar sustentável para todos.

A definição brasileira de Segurança Alimentar e Nutricional (SAN), formulada pelo Fórum Brasileiro de Segurança Alimentar e Nutricional (FBSAN), aprovada na II Conferência Nacional de Segurança Alimentar e Nutricional (2004) e, posteriormente, incorporada na Lei Orgânica de Segurança Alimentar (LOSAN):

Segurança Alimentar e Nutricional (SAN) é a realização do direito de todos ao acesso regular e permanente a alimentos de qualidade, em quantidade suficiente, sem comprometer o acesso a outras necessidades essenciais, tendo como base práticas alimentares promotoras de saúde, que respeitem a diversidade cultural e que sejam social, econômica e ambientalmente sustentáveis. (CONSEA, 2004).

Muitos movimentos sociais são envolvidos ao se tratar de programas alimentares, alguns deles ligados à reforma agrária, vão contra a ideia que se impõe à produção agropecuária de alguns países, onde não se produz para quem tem fome, mas para quem tem dinheiro. O conceito de SAN, por sua vez, vai contra essa realidade, e afirma que é possível estruturar uma política nacional, como está ocorrendo no Brasil: a construção de uma política de Estado (CUSTODIO, et al, 2011).

O sistema governamental, deve implementar cada vez mais, modos de abastecer, comercializar e consumir alimentos, tendo em vista a sustentabilidade socioeconômica e ambiental, respeitando a diversidade cultural e promovendo a saúde no sentido do direito humano à alimentação saudável e adequada. SAN não é apenas a disponibilidade do alimento, é acima de tudo a permanência ao acesso de alimento com qualidade. A noção de segurança socioeconômica, qualidade sanitária do alimento e respeito ao meio

Segurança alimentar. Revista Revise, vol 3, no fluxo contínuo, p.46-63. 

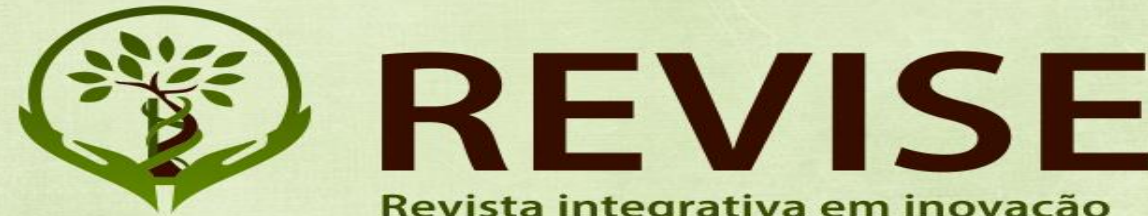

Revista integrativa em inovação tecnológica nas ciências da saúde

ISSN: $2179-6572$

ambiente, geram a estabilidade ou a segurança com o alimento, dessa forma, pode estabelecer o conceito de segurança alimentar e nutricional, que transita nas esferas biológica, econômica, social, ambiental e cultural (FREITAS, 2007).

Corrobora ainda que o conceito de segurança alimentar e nutricional no Brasil remete à necessidade do entendimento de questões estruturais, em que a desigualdade social influi muito na questão. Como uma expressão que se movimenta em várias áreas do conhecimento, a SAN pode representar proposições para situações específicas e conjugadas às necessidades biológicas e sociais dos indivíduos.

A política estratégica para a SAN está relacionada ao acesso universal ao alimento e às políticas sociais de assistência à população, essas políticas devem cumprir a função no âmbito dos direitos sociais. Portanto, os projetos em SAN, mesmo os transitórios, de caráter assistencialista, como campanhas fundadas na tradição da caridade, mantêm-se flexíveis às políticas econômicas. A SAN remete à importância do controle social em torno de programas sociais de saúde e alimentação e via conselhos, que devem ser atuantes para a melhoria da qualidade de vida, educação, saúde e nutrição.

Infelizmente, saúde e nutrição nem sempre são prioridades práticas de políticas locais no Brasil, os significados sobre o cuidado da alimentação se confundem e são delimitados por estilos particulares, fator este que pode ser influenciado pela cultura, caracterizados pela ausência de uma organização social capaz de qualificar o programa social de alimentação e nutrição e redefinir, em primeira instância, critérios para a SAN (BRASIL, 2013).

Segundo Belick (2003) as políticas de Segurança Alimentar e Nutricional são essenciais para garantir o acesso aos alimentos para os menos favorecidos, atendendo as extensões da quantidade e qualidade dos produtos ofertados, devendo garantir que as pessoas possam se alimentar com cidadania. $\mathrm{O}$ autor afirma que o principal problema brasileiro está na falta de poder aquisitivo por uma boa parte da população no que diz respeito à manutenção da sua sobrevivência, indo ao contrário de outros países, o Brasil

Segurança alimentar. Revista Revise, vol 3, no fluxo contínuo, p.46-63. 

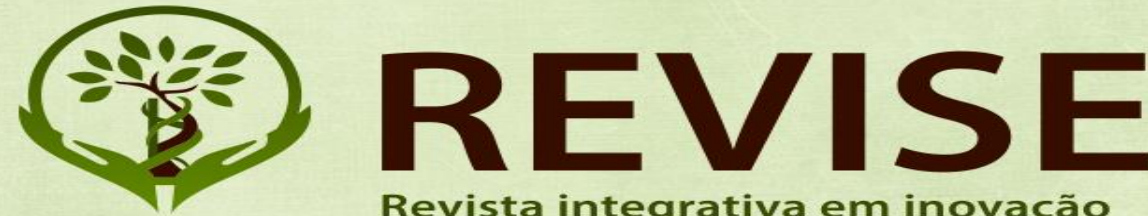

Revista integrativa em inovação tecnológica nas ciências da saúde

ISSN: 2179-6572

tem uma boa oferta de alimentos, porém, não são acessíveis à população de renda mais baixa.

Desta forma a SAN, se aproxima do enfoque da "nova promoção da saúde", que não só realiza a crítica em relação à visão preventiva da saúde, mas também se propõe a atuar, visando a criação de fatores favoráveis à saúde (OLIVEIRA, 2005).

\subsection{POLÍticas PÚBLICAS DE ALIMENTAÇÃO E NUTRIÇÃO NO} BRASIL: PNAN e PNAE

\subsubsection{Programa nacional de alimentação escolar - PNAE}

O Brasil tem uma vasta experiência na implementação de programas sociais voltados para a questão alimentar e nutricional, como por exemplo, o Programa Nacional de Alimentação Escolar (PNAE), a Política Nacional de Alimentação e Nutrição (PNAN), dentre outros. A construção de políticas públicas que garantam os direitos do cidadão deve fazer parte da agenda dos que estão comprometidos com a educação, com a consolidação da democracia e com o combate às injustiças da sociedade contemporânea. Dessa forma, em 1995, surge a alimentação escolar, uma política inicialmente compensatória, que se constitui, atualmente, como a garantia universal do direito humano à alimentação. Antes de sua institucionalização, registros mostram que essa política já era utilizada em algumas comunidades, sendo custeada por sociedades filantrópicas, porém o poder público aos poucos, foi dando suporte às ações de alimentação ao escolar, criando, em 1955, o programa que atualmente é conhecido como Programa Nacional de Alimentação Escolar (CHAVES, et al, 2009).

O PNAE tem como objetivo contribuir para o crescimento e o desenvolvimento biopsicossocial, a aprendizagem, o rendimento escolar e a formação de práticas alimentar saudáveis dos alunos, por meio de ações de educação alimentar e nutricional e da oferta de refeições que cubram as suas necessidades nutricionais durante o período letivo (BRASIL, 2013).

Segurança alimentar. Revista Revise, vol 3, no fluxo contínuo, p.46-63. 

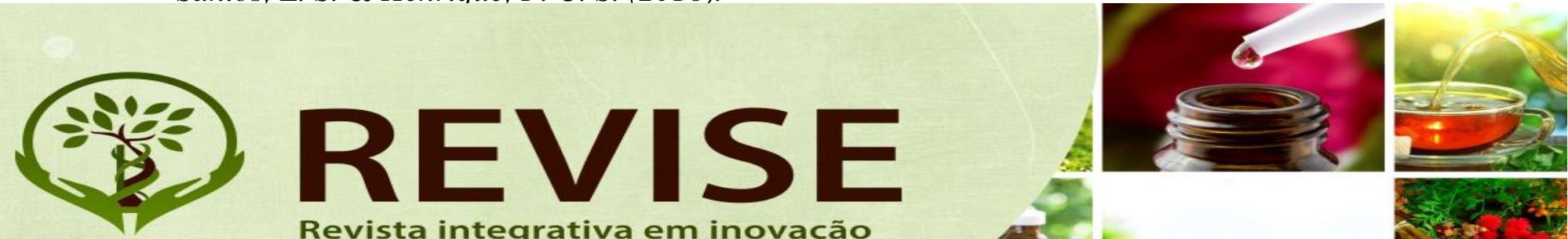

Revista integrativa em inovação tecnológica nas ciências da saúde

Segundo a resolução de $n^{\circ} 26$ do FNDE, e tendo como fundamentação legal a lei, 11.947, adota algumas diretrizes, sendo elas: o emprego da alimentação saudável e adequada, compreendendo o uso de alimentos variados, seguros e que respeitem a cultura, a inclusão da educação alimentar e nutricional no processo de ensino e aprendizagem, o apoio ao desenvolvimento sustentável, a participação da comunidade no controle social, o direito à alimentação escolar, visando garantir a segurança alimentar e nutricional dos alunos. A lei garante que serão atendidos pelo PNAE os alunos matriculados na educação básica das redes públicas. Além das diretrizes, foram adotados alguns princípios para que regem esta política, sendo eles: equidade, participação social, universalidade, continuidade, compartilhamento de decisões, direito humano a alimentação adequada e respeito a cultura (BRASIL, 2010).

O Programa funciona por meio da transferência de recursos financeiros em caráter suplementar, de forma a garantir, no mínimo, 15\% das necessidades diárias dos alunos do ensino infantil e fundamental e 30\% das necessidades diárias dos alunos das creches, das escolas indígenas e daquelas localizadas em áreas remanescentes de quilombos. Tem como objetivo atender às necessidades nutricionais dos alunos e contribuir para a formação de hábitos alimentares saudáveis durante sua permanência em sala de aula, colaborando para o seu crescimento, desenvolvimento, aprendizagem e rendimento escolar (BRASIL, 2010).

O PNAE está inserido na Política Nacional de Segurança Alimentar e Nutricional desde 2005, sendo uma das políticas intersetoriais que garantem acesso aos alimentos. Com a intenção de incentivar a formação de hábitos alimentares saudáveis, o PNAE traz alguns princípios, o respeito aos hábitos alimentares saudáveis, as práticas tradicionais que fazem parte da cultura e da preferência alimentar local, dessa forma, o Programa contribui para a preservação dos hábitos alimentares regionais (BRASIL, 2006).

A Coordenação Geral do PNAE, visando a melhoria dos seus objetivos, diretrizes e das suas estruturas passou a chamar-se como programa de alimentação 

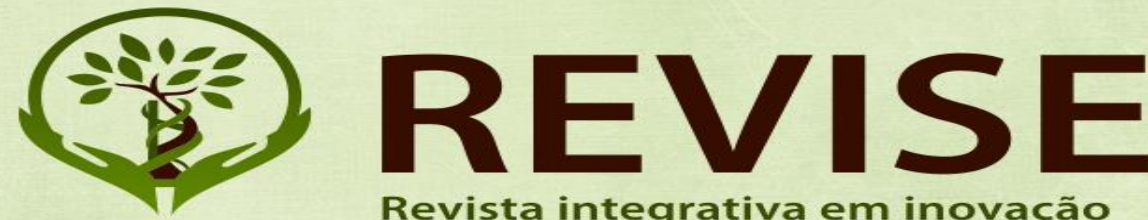

Revista integrativa em inovação tecnológica nas ciências da saúde

ISSN: 2179-6572

escolar saudável para rede pública de educação básica de jovens e adultos, e também passou a estabelecer que no mínimo 30\% dos repasses do PNAE fossem investidos na aquisição de produtos da agricultura familiar. Sendo assim, de acordo com as políticas públicas e dos movimentos sociais de caráter universal, o PNAE passou a congregar em suas estratégias de execução os seguintes princípios para a gestão e a execução da alimentação escolar: equidade, participação social, universalidade, sustentabilidade, continuidade, compartilhamento de responsabilidades, direito humano à alimentação adequada e respeito aos hábitos e tradições regionais (LIMA, 2010).

A promoção da saúde no ambiente escolar vem sendo fortemente e reforçada por órgãos internacionais, essa promoção é recomendada pelo PNAE, pois este abrange uma grande cobertura alimentar no País, cerca de 36 milhões de estudantes desfrutam dessa promoção no próprio ambiente escolar. O PNAE é conhecido mundialmente como um caso de sucesso de Programa de alimentação escolar sustentável. Nesse contexto é importante ressaltar os acordos Internacionais firmados com a Organização das Nações Unidas para alimentação e agricultura - FAO, e com o Programa Mundial de Alimentos - PMA, por meio da Agência Brasileira de Cooperação do Ministério das Relações Exteriores sob os princípios da SAN e da DHAA (BRASIL, 2014, P. 10).

Em 17 de Junho de 2013, foi publicada a Resolução FNDE nº 26, que fortalece um dos eixos do programa, a Educação Alimentar e Nutricional (EAN). Essa medida vai ao encontro das políticas públicas atuais relacionadas a SAN (BRASIL, 2014). Assim sendo, é de suma importância que as escolas forneçam um cardápio que seja capaz de atender às necessidades nutricionais dos alunos durante sua permanência em sala de aula, contribuindo para a preservação e o resgate da cultura alimentar brasileira (CHAVES, 2009).

\subsection{Política nacional de alimentação e nutrição - PNAN}

Segurança alimentar. Revista Revise, vol 3, no fluxo contínuo, p.46-63. 

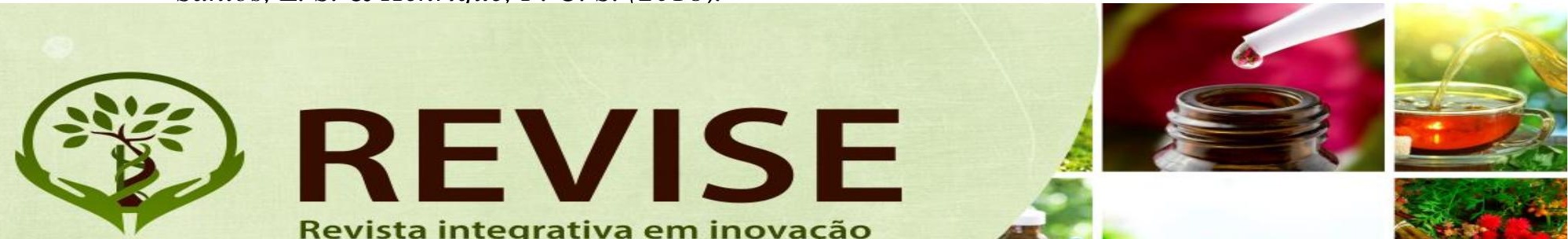

Revista integrativa em inovação tecnológica nas ciências da saúde

Em 1999, o Ministério da Saúde tomou uma série de medidas importantes, sendo uma delas a institucionalização da Política Nacional de Alimentação e Nutrição, PNAN, aprovada pela Portaria Ministerial $n^{\circ} 710 / 1999$, como parte integrante da Política Nacional de Saúde, inserindo-se no contexto da Segurança Alimentar e Nutricional. A formulação e implementação dessa Política foi de responsabilidade da Secretaria de Políticas de Saúde, tendo também o apoio de diferentes setores do governo.

Para assegurar os direitos humanos no âmbito da alimentação e nutrição, a definição dessa política setorial compreendeu a revisão de conceitos, levando em conta a diversidade, e a necessidade de tratamento diferenciado, e tendo por base a análise da situação, alimentar e nutricional da população. Essa revisão implicará, por via de consequiência, o redimensionamento das práticas, mediante a formulação ou readequação dos planos, programa, projetos ou atividades, que operacionalizarão as diretrizes fixadas nesta Política Nacional (BRASIL, 2007, p. 17).

Com foco nas transições demográficas, epidemiológicas e nutricional, a aprovação da PNAN representou um marco na história da SAN. Desde então, a escassez de alimento e a fome, vem diminuindo, através de políticas de alimentação e Nutrição. A adoção dessa Política representa um marco importante na medida em que a alimentação e a nutrição são condições básicas para a promoção e a proteção da saúde. No Brasil se convive com situações extremas: a desnutrição e a carência de alguns nutrientes essenciais e as altas e crescentes taxas de obesidade. Essa Política adentra no contexto da segurança alimentar e nutricional e tem como objetivos garantir a qualidade dos alimentos que são ofertados para consumo no país, a promoção de práticas alimentares saudáveis e a prevenção e o controle dos distúrbios nutricionais, bem como o estímulo às ações intersetoriais que propiciem o acesso dos alimentos. A PNAN traz um modelo da SAN, baseado no direito humano à alimentação, onde salienta que a alimentação e a nutrição de qualidade, são requisitos de promoção e proteção da saúde (RECINE, 2010).

Segurança alimentar. Revista Revise, vol 3, no fluxo contínuo, p.46-63. 

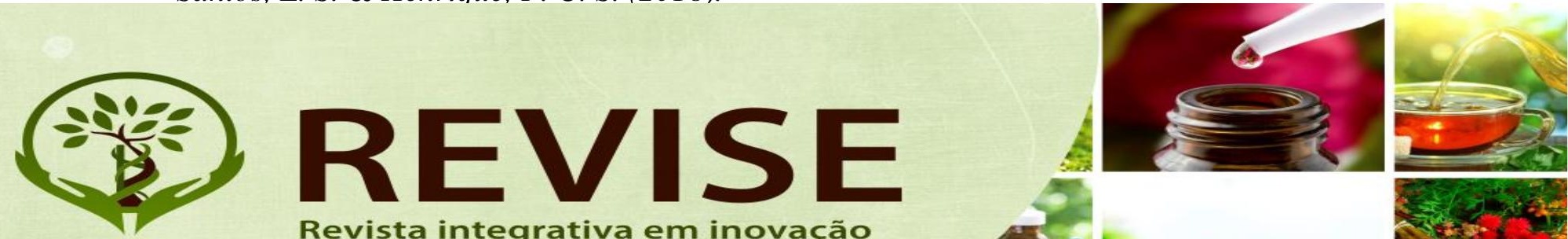

Revista integrativa em inovação tecnológica nas ciências da saúde

A Política Nacional de Alimentação e Nutrição, integra a Política Nacional de Saúde, inserindo-se ao mesmo tempo, no contexto de Segurança Alimentar e Nutricional. Dessa forma dimensionada - e compondo, portanto, o conjunto de políticas do governo voltadas a concretização do direito humano universal à alimentação a nutrição adequadas (BRASIL, 2007, p. 17).

De acordo com o discurso, a PNAN, com o estímulo às ações intersetoriais, tem o dever de garantir a qualidade dos alimentos consumidos no país, promover práticas alimentares saudáveis, prevenir e controlar distúrbios nutricionais. Para isso, algumas diretrizes foram expressas na PNAN, mas especificamente sete diretrizes essenciais, sendo elas: estímulo às ações intersetoriais com vistas ao acesso universal aos alimentos; garantia da segurança e da qualidade dos alimentos e da prestação de serviços neste contexto; monitoramento da situação alimentar e nutricional; promoção de práticas alimentares e estilos de vida saudáveis; prevenção e controle dos distúrbios nutricionais e de doenças associadas à alimentação e nutrição; promoção do desenvolvimento de linhas de investigação; e desenvolvimento e capacitação de recursos humanos.

Com a aprovação da PNAN, fez-se necessário que houvesse um processo permanente de capacitação de recursos humanos envolvidos em atividades de planejamento, monitoramento e avaliação, esse processo tomou como base a segurança alimentar nutricional e direito humano à alimentação. Nas últimas décadas, tem-se visto algumas evoluções nas ações do Ministério da Saúde, programas mais amplos foram implementados, como, Programa Bolsa Alimentação, Bolsa Família. Outro mérito que deve ser atribuído ao PNAN foi a implantação do SISVAN, o qual tem a finalidade de reunir informações facilitando políticas públicas para a melhoria das condições nutricionais da população e a manutenção de um eixo de convergência setorial importante na Saúde Pública. (CARVALHO, 2011).

Como toda política nacional, a PNAN é de responsabilidade dos gestores, onde, considerando os princípios do SUS, de forma articulada e devem viabilizar o alcance do 

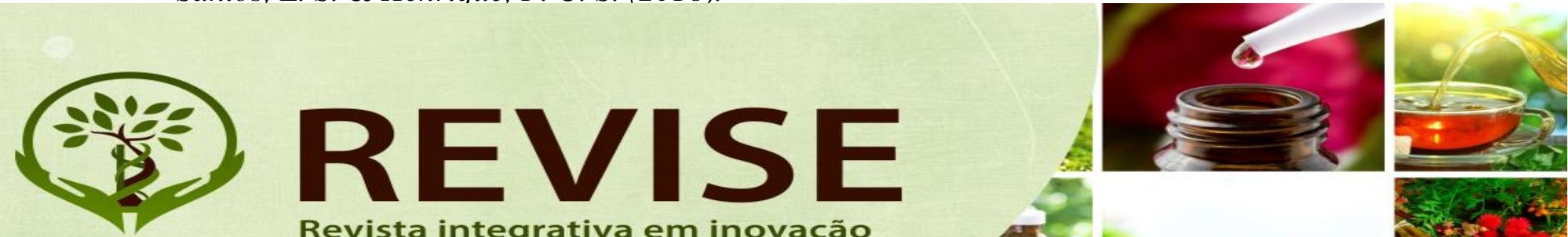

Revista integrativa em inovação tecnológica nas ciências da saúde

ISSN: 2179-6572

propósito desta política. Devem considerar as características peculiares de intersetorialidade e vinculação com a SAN (BRASIL, 2007).

A busca de parceria com os demais setores envolvidos na Segurança Alimentar Nutricional, e por conseguinte, na concretização do direito humano à alimentação e nutrição adequada, levará em conta a adoção ou implementação de medidas que poderão redundar em impacto importante sobre a saúde da população (BRASIL, 2007, p. 30).

A alimentação, nutrição e a vigilância devem formar um conjunto quando se trata da Promoção da Saúde enquanto prioridade do Ministério da Saúde, a alimentação e nutrição sempre fizeram parte das preocupações do país, embora alterasse o nível de prioridade ou a competência dos governos ao lidarem com tanta complexidade referente à questão. O processo de construção da PNAN constituiu-se em um enorme aprendizado, desencadeando da formulação de outras políticas públicas no âmbito do Ministério da Saúde.

\section{4- CONCLUSÃO}

É possível perceber que a SAN passou a ocupar um espaço central nas estratégias de desenvolvimento humano, sendo que central à promoção de inclusão econômica e social da população nos meios de produção, promoção da soberania alimentar, melhoria da qualidade de vida, preservação da cultura e meio ambiente. Porém, com todo esse avanço, a SAN ainda está muito distante de ser uma realidade presente na vida de muitos.

Através da SAN, foi possível aumentar o quadro dos programas sociais, dentre eles os mais conhecidos, Programa de Aquisição de Alimentos - PAA; Programa Nacional da Alimentação do Escolar - PNAE; Programa Bolsa Família - PBF; Programa Nacional da Reforma Agrária (MDA); Política Nacional de Alimentação e Nutrição - PNAN; dois desses foram parte da discussão deste trabalho e esses 

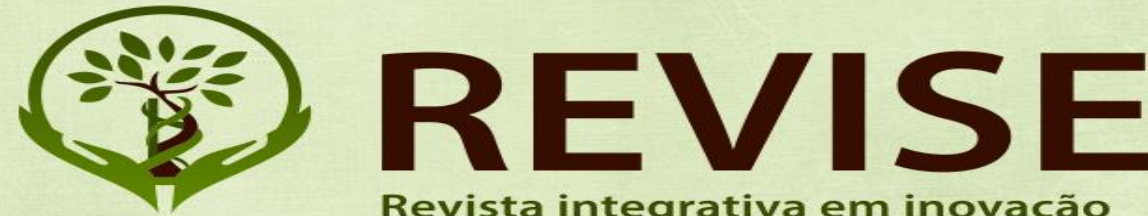

Revista integrativa em inovação tecnológica nas ciências da saúde

ISSN: 2179-6572

programas possibilitam o reconhecimento de que a população em situação de risco é maior do que a normalmente identificada.

Ao realizar esse estudo, pode-se perceber que as políticas intersetoriais estão realmente inseridas no contexto da $\mathrm{SAN}$, e se aproximam realmente do contexto de segurança alimentar, essa ideia fica explícita dentro de suas ações e diretrizes, onde sempre estão voltadas para o fornecimento de alimentos de uma forma segura e visando alcançar um bom estado nutricional e a erradicação da fome.

Concluiu-se também que o processo de avaliação política é de fundamental importância para o avanço da continuidade das ações respaldadas para a consolidação do PNAN, como política setorial da saúde promotora, por meio das ações do SUS e da SAN. As políticas avaliadas são orientadas pelo princípio da intersetorialidade, que é uma garantia da SAN, desta forma, elas inserem-se na saúde o debate da SAN. Essas políticas são de trajetória fundamental para a SAN, pois articulam ações que reafirmam os valores da promoção da saúde e da cidadania.

Sabe-se que toda política é de extrema importância para a melhoria das condições de sobrevivência do ser humano, as citadas aqui são de importância impar para erradicar a fome, diminuir o índice de pobreza, porém em alguns aspectos ainda se demonstra frágil, o que remete a pensar que ainda exige algumas melhorias, como realmente garantir o acesso ao alimento a todos, que é uma realidade que infelizmente não acontece e uma melhoria nos alimentos ofertados em muitas escolas no Brasil, e isso cabe aos gestores e a fiscalização da população.

\section{REFERÊNCIAS}

ALMEIDA, Daniela Lima de. (2010). Discente da Unifor: Alimentação adequada como direito fundamental: desafios para garantir a efetivação. 2010. Artigo submetido ao II Concurso de artigos jurídicos.

BARDIN, L. (1979) Análise de conteúdo. Lisboa: Edições 70, 229 p.

MARCONE, M. A.; LAKATOS, E.M. (2009.) Fundamentos da Metodologia Científica. 6 ed. São Paulo: Editora Atlas, P. 185. 

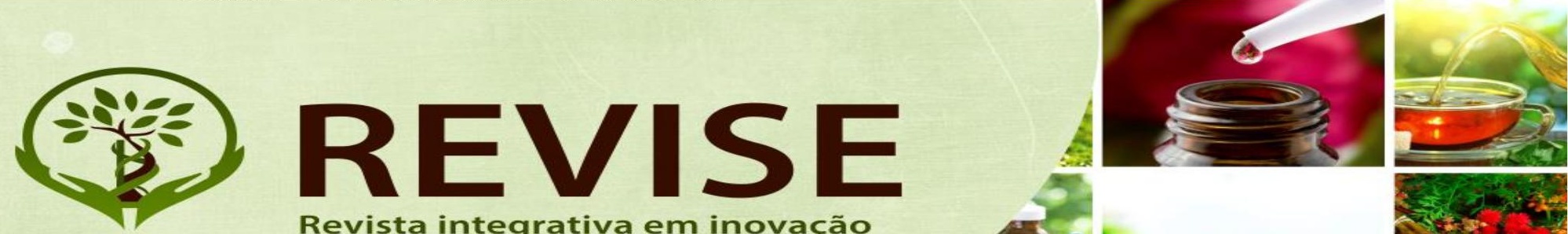

Revista integrativa em inovação tecnológica nas ciências da saúde

BELIK, Walter. (2013) Perspectivas para segurança alimentar e nutricional no Brasil. Saúde e Sociedade, São Paulo, v. 1, n. 12, p.2-20.

BRASIL. Fundo Nacional de Desenvolvimento da Educação(2012). Programa Nacional de Alimentação Escolar. Brasília/DF,

- Departamento de atenção básica.(2012). Política Nacional de Alimentação e Nutrição. Brasília/DF,

Coordenação Geral de Alimentação e Nutrição(2010). Sistema Nacional de Vigilância Alimentar e Nutricional. Brasília, DF, Disponível em:

http://nutricao.saude.gov.br/sisvan.php.

BRASÍLIA, Tribunal de Contas da União. (2010).Cartilha para Conselheiros do Programa Nacional de Alimentação Escolar (PNAE). $5^{\text {a }}$ ed. Brasília

BURLANDY, Luciene. (2009). A construção da política de segurança alimentar e nutricional no Brasil: estratégias e desafios para a promoção da intersetorialidade no âmbito federal de governo.Ciênc. saúde coletiva, Rio de Janeiro, v. 14, n.3.

BURLANDY, Luciene.( 2008). Segurança alimentar e nutricional e saúde pública. Cad. Saúde Pública, Rio de Janeiro, 24(7):1464-1465, . Disponível em http://www.scielo.br/pdf/csp/v24n7/01.pdf\&gt;>

CHAVES, et al. (2009). Políticas de alimentação e nutrição: Brasil e Portugal. Revista da Sociedade Portuguesa de Ciências da Nutrição e Alimentação, Porto, v. 16, n. 1, p. 3-1.

CONSEA - CONSELHO NACIONAL DE SEGURANÇA ALIMENTAR E NUTRICIONAL. (2004). II Conferência Nacional de Segurança Alimentar e Nutricional. A construção da Política Nacional de Segurança Alimentar e Nutricional. Relatório final. Olinda, PE: Consea.

CUSTÓDIO, Marta Battaglia et al. (2011). Segurança Alimentar e Nutricional e a construção de sua política: uma visão histórica. Revista Saúde, Campinas, v. 5, n. 8, p.1-10. 

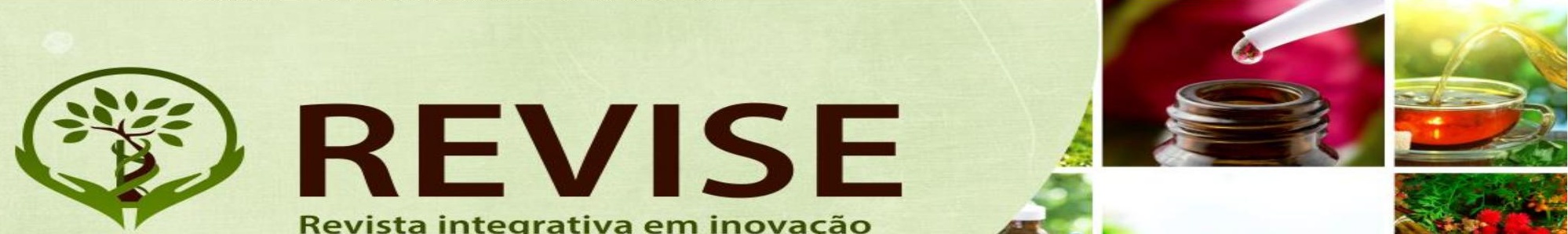

Revista integrativa em inovação tecnológica nas ciências da saúde

CUSTÓDIO, MB. (2009). Política Nacional de Segurança Alimentar e Nutricional no Brasil: Arranjo Institucional e Alocação de Recursos [tese]. São Paulo: Programa Interunidades em Nutrição Humana Aplicada, Universidade de São Paulo;

FAO - FOOD AND AGRICULTURE ORGANIZATION. Voluntary guidelines to support the progressive realization of the right adequate food in the context of national food security.

FREITAS, Maria do Carmo Soares de; PENA, Paulo Gilvane Lopes. (2007). Segurança Alimentar e nutricional: Uma Produção do Conhecimento com ênfase em Aspectos da Cultura. Rev. Nutr. , Campinas, v 20, n. 1. Disponível em: http://www.scielo.br/scielo.php?script=sci_arttext\&amp;pid=S1415-5

2732007000100008\&amp;lng=en\&amp;nrm=iso\&gt;> . Acesso em 13 de maio de 2014.

MINAYO, M. C. S. O. (2010). Latino-americano e do Caribe em Ciências da Saúde ID: Desafio do conhecimento: pesquisa qualitativa em saúde. 12. Ed. São Paulo: Hucitec.

Ministério do Desenvolvimento Social e Combate à Fome. (2010).

Secretaria Nacional de Segurança Alimentar e Nutricional - SESAN. Brasília/DF.

Ministério da Saúde.( 1986). Relatório da VIII Conferência Nacional de Saúde. Brasília: Ministério da Saúde.

Ministério da Saúde(2011b). Bolsa Família na saúde. Brasília, DF.

OLIVEIRA, Y. P. (2005). Suplementação Alimentar e Combate a Carências Nutricionais Específicas - Subsídios Básicos, Brasília.

PRADO, Shirley Donizete et al. (2010). A pesquisa sobre segurança alimentar e nutricional no Brasil de 2000 a 2005: tendências e desafios. Ciênc. Saúde coletiva, Rio de Janeiro.

Segurança alimentar. Revista Revise, vol 3, no fluxo contínuo, p.46-63. 